Biotempo (Lima)

ORIGINAL ARTICLE / ARTÍCULO ORIGINAL

PRACTICAL CONJUGATION FOR THE FORMATIVE EVALUATION OF LEARNING

\title{
CONJUGACIÓN PRÁCTICA PARA LA EVALUACIÓN FORMATIVA DEL APRENDIZAJE
}

\section{Cecilia G. Solano-García ${ }^{1}$; George Argota-Pérez ${ }^{2}$, Carmen L. Chauca-de-Quispe ${ }^{1}$; Josefa B. Pari- Olarte $^{3} \&$ Eusebio Aliaga-Guillen ${ }^{4}$}

1 Facultad de Odontología. Universidad Nacional “San Luis Gonzaga”. Ica, Perú.

E-mail: cecisoga@gmail.com; carmen_chauca@hotmail.com

2 Centro de Investigaciones Avanzadas y formación Superior en Educación, Salud y Medio Ambiente "AMTAWI", Puno-Perú. E-mail: george.argota@gmail.com

3 Facultad de Bioquímica y Farmacia. Universidad Nacional “San Luis Gonzaga”. Ica, Perú. E-mail: berthapari@ hotmail.com

4 Facultad de Medicina Humana. Universidad Nacional “San Luis Gonzaga”. Ica, Perú.

E-mail: chevokid@hotmail.com

Author for correspondence: george.argota@gmail.com

\begin{abstract}
The search for strategies for learning represents a challenge at the educational level. The purpose of the study was the practical conjugation for the formative evaluation of learning in university teachers. From the implementation of the graduate program referred to the international diploma course "scientific creativity and technological innovation" that was developed at the National University "San Luis Gonzaga" of Ica, Peru, a learning scheme was established on 6 indicators: selection variable, research approach, type of scientific article oriented, cognitive recognition, methodological structure of interest and interpretation, and contribution criterion. Three teachers were randomly selected, and through the quantitative technique by content analysis the level of learning was considered. A score code of 12 to 20 was established, with only the 18 to 20 points being recognized as the level of excellence. When comparing the results among the teachers, there were statistically significant differences $(p<0.05)$ where a teacher ranked at the level of excellence when obtaining 18 points. The replication of the indicators in other teaching modalities was required to evaluate their possible contribution to the formation of learning in university teachers.
\end{abstract}

Keywords: formative evaluation - higher education - instructional process - teaching - learning 


\section{RESUMEN}

La búsqueda de estrategias para el aprendizaje representa un desafío en el nivel educativo. El propósito del estudio fue la conjugación práctica para la evaluación formativa del aprendizaje en docentes universitarios. A partir de la implementación del programa de posgrado referido al diplomado internacional "creatividad científica e innovación tecnológica" que se desarrolló en la Universidad Nacional "San Luis Gonzaga" de Ica, Perú se estableció un esquema de aprendizaje sobre 6 indicadores: variable de selección, enfoque de la investigación, tipo de artículo científico orientado, reconocimiento cognoscitivo, estructura metodológica de interés y criterio de interpretación y aporte. Fueron seleccionados de forma probabilística aleatoria, 3 docentes y mediante la técnica cuantitativa por análisis de contenido se consideró el nivel sobre el aprendizaje. Se estableció un código de puntuación de 12 a 20 siendo reconocido solamente como nivel de excelencia el rango de 18 a 20 puntos. Al comparar los resultados entre los docentes, hubo diferencias estadísticamente significativas $(\mathrm{p}<0,05)$ donde un docente se ubicó en el nivel de excelencia al obtener 18 puntos. Se requirió, la replicación de los indicadores en otras modalidades de la enseñanza para evaluar su posible contribución a la formación del aprendizaje en docentes universitarios.

Palabras clave: aprendizaje - educación superior - enseñanza - evaluación formativa - proceso de instrucción

\section{INTRODUCCIÓN}

En la actualidad, el uso de dispositivos está siendo común para el aprendizaje y bienestar (Dimitrov, 2016). Sin embargo, algunas brechas en el comportamiento y respuesta durante el proceso de aprendizaje, es consecuencia de incorrectas o poca atención a las prácticas en la evaluación formativa (EF). Con base posterior en sólidas preparaciones la $\mathrm{EF}$ proporciona, carácter de retroalimentación entre profesores y estudiantes posibilitando guiar de forma efectiva, el proceso de instrucción (Black \& Wiliam, 2009), además, indica la significación del aprendizaje (Sach, 2012; Suurtamm \& Koch, 2014).

Lisle (2015) expresa que, la medición continua provee la propia evaluación formativa, pues durante la sumatoria de calificaciones se garantiza la preparación de los estudiantes. En este sentido, Carless (2011) y Yin \& Buck (2015) señalan como razón esencial para el logro de la EF, realizar prácticas sistemáticas y donde la responsabilidad de su implementación miden el propio aprendizaje como único recurso de validez (NRC, 2014). La EF conduce a la investigación formativa (IF) como proceso de aprendizaje para el desarrollo cultural de los estudiantes sobre la investigación, independientemente de cualquier resultado científico pretendido a obtenerse, pues su objetivo es la creación de habilidades, motivación y análisis crítico que faciliten fortalecer las capacidades profesionales con vínculo hacia la investigación (Facundo et al., 2007; Tejada et al., 2008). La formación, es la evaluación sistemática para el logro del aprendizaje de acuerdo a, objetivos programados o requisitos que deben cumplir y esto representa una premisa del beneficio en la educación (Gikandi et al., 2011; Llamas et al., 2013; Tarighat \& Khodabakhsh, 2016). Considerando que, la educación como nivel de transmisión y formación está bien estructurado, independientemente de cuánto puede diferenciarse de forma positiva o no por el uso de las tecnologías de la información y las comunicaciones (Yilmaz et al., 2017; Vo et al., 2017), los estudiantes necesitan algunas orientaciones, procedimientos $\mathrm{o}$ aplicativos para garantizar sus conocimientos.

El propósito del estudio fue la conjugación práctica para la evaluación formativa del aprendizaje en docentes universitarios.

\section{MATERIALES Y MÉTODOS}

El estudio se realizó en la Universidad Nacional "San Luis Gonzaga“ de Ica, Perú durante el 2018. Se consideró durante el programa de diplomado internacional "Creatividad Científica e Innovación Tecnológica", la búsqueda sobre esquemas evaluativos para la formación del aprendizaje en docentes universitarios y que estuviera basada con la tenencia cultural relacionada a la metodología de la investigación científica.

Con base al modelo (figura 1) se estableció un esquema de aprendizaje sobre 6 indicadores: variable de selección, enfoque de la investigación, tipo de artículo científico orientado, reconocimiento cognoscitivo, estructura 
metodológica de interés y criterio de interpretación y aporte. Los 5 primeros indicadores se le asignó un código de puntuación de 3 mientras que, el último fue de 5 puntos. La sumatoria total de puntos fue de 20 reconociendo solo el rango de 18 a 20 como de excelencia. Fueron seleccionados 3 docentes de forma probabilística aleatoria y mediante la técnica cuantitativa por análisis de contenido para considerar el nivel sobre el aprendizaje.
Para el análisis de los resultados se utilizó el programa estadístico profesional Statgraphics Centurion 18 donde la normalidad de los datos fue mediante la prueba Kolmogorov-S. Los datos se compararon por análisis de la varianza siendo el contraste múltiple de rango analizado por la prueba de Bonferroni y donde se consideró significativo los resultados cuando $\mathrm{p}<0,05$.

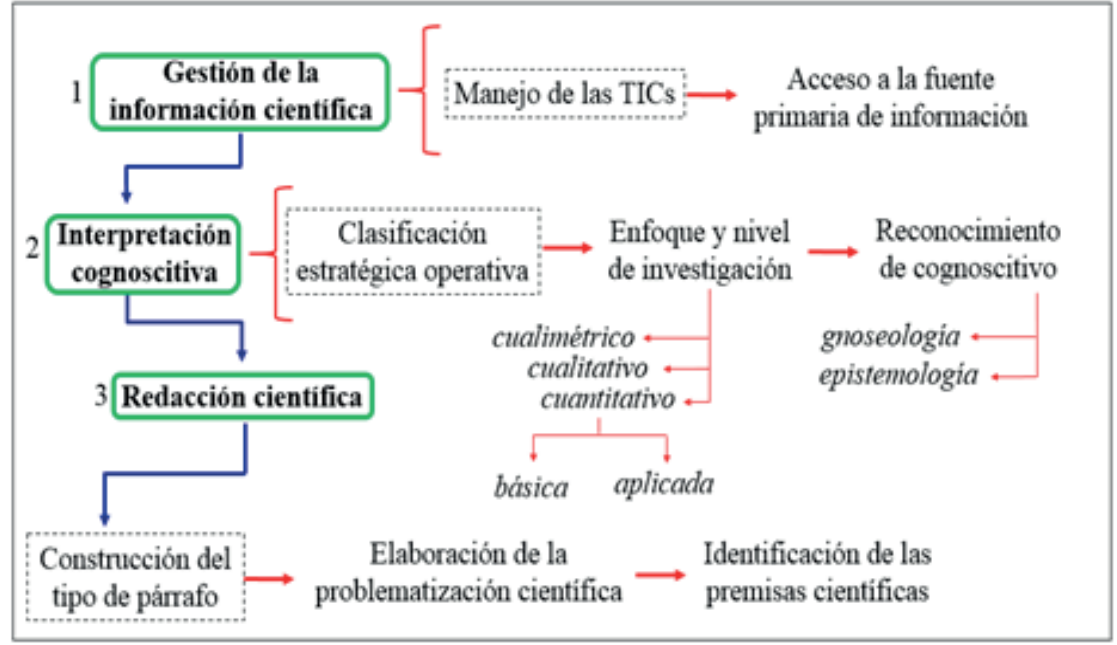

Figura 1. Modelo cognitivo metodológico para la generación y aportes de resultados científicos.

\section{RESULTADOS}

La tabla 1 muestra, la conjugación de la práctica para la evaluación formativa del aprendizaje en docentes universitarios.

Tabla 1. Conjugación práctica / evaluación formativa del aprendizaje.

\begin{tabular}{cccccc}
\hline $\begin{array}{l}\text { Variable de } \\
\text { selección }\end{array}$ & $\begin{array}{c}\text { Enfoque de la } \\
\text { investigación }\end{array}$ & $\begin{array}{c}\text { Tipo de artículo } \\
\text { científico orientado }\end{array}$ & $\begin{array}{c}\text { Reconocimiento } \\
\text { cognoscitivo }\end{array}$ & $\begin{array}{c}\text { Estructura } \\
\text { metodológica de } \\
\text { interés }\end{array}$ & $\begin{array}{c}\text { Criterio de } \\
\text { interpretación y } \\
\text { aporte }\end{array}$ \\
\hline
\end{tabular}

La tabla 2 muestra, la información comunicada por los docentes donde se encontró diferencias estadísticamente significativas $(\mathrm{p}<0,05)$ según el código de puntuación obtenido (tabla 3 ).

Tabla 2. Código de puntuación / docentes evaluados.

\begin{tabular}{ccccccc}
\hline Indicador & Docente 1 & CP & Docente 2 & CP & Docente 3 & CP \\
\hline Variable de selección & $\mathrm{x}$ & 3 & $\mathrm{y}$ & 3 & $\mathrm{z}$ & 3 \\
Enfoque de la investigación & Cuantitativo & 3 & Cuantitativo & 3 & Cuantitativo & 3 \\
Tipo de artículo científico orientado & Opinión & 3 & Original & 3 & Reporte de caso & 3 \\
Reconocimiento cognoscitivo & Gnoseología & 3 & Gnoseología & 3 & Epistemología & 3 \\
Estructura metodológica de interés & Objeto & 3 & Campo & 3 & Matriz & 3 \\
Criterio de interpretación y aporte & modificación & 0 & Aceptado con & 3 & Aceptado con & 2 \\
Total de puntuación & & & modificación & & modificación & \\
& & & & 15 & & 17 \\
\hline
\end{tabular}


Tabla 3. Análisis de varianza / prueba de contraste múltiple. $\mathrm{FV}=$ Fuente de variación. $\mathrm{SC}=$ Suma de cuadrados. $\mathrm{gl}=$ grados de libertad. $\mathrm{CM}=$ Cuadrado medio. $\mathrm{F}=$ Estadístico de Fisher. $\mathrm{P}=$ Probabilidad o significancia.

\begin{tabular}{|c|c|c|c|c|c|}
\hline FV & SC & $\mathrm{gL}$ & $\mathrm{CM}$ & Coeficiente F & Valor P \\
\hline Entre grupos & 13,14 & 2 & 6,57 & 58,01 & 0,0001 \\
\hline Intra grupos & 0,68 & 6 & 0,11 & & \\
\hline Total (Corr.) & 13,82 & 8 & & & \\
\hline Docente & Grupos H & & & & \\
\hline Docente 1 & $\mathrm{x}$ & & & & \\
\hline Docente 3 & $\mathrm{x}$ & & & & \\
\hline Docente 2 & $\mathrm{x}$ & & & & \\
\hline
\end{tabular}

\section{DISCUSIÓN}

La formación mediante el aprendizaje permanente, es un reto actual para la educación (Iu et al., 2014) donde la búsqueda de estrategias conscientes en los propios procesos cognitivos deben evaluarse para el reconocimiento diferenciado del aprendizaje (Cross, 2015) de modo que, cualquier introducción práctica innovativa para satisfacer las expectativas y no continuar con enfoques tradicionales, resulta de interés (Messum et al., 2016; Jackson, 2016).

En este estudio, se midieron 6 indicadores donde se observó coincidencia solo en uno de ellos: el enfoque de la investigación (cuantitativo). Los docentes alcanzaron el máximo de puntuación excepto en el indicador: criterio de interpretación y aporte donde uno de ellos incluso, no obtuvo calificaciones debido a, sugerencias por modificar mientras que otro, resultó ubicarse en el nivel de excelencia establecido (18-20 puntos). En este criterio debe conjugarse el reconocimiento cognoscitivo con los valores potenciales de la investigación: conveniencia social, relevancia social, económica, ambiental, normativa, científica o tecnológica, implicaciones prácticas, valor teórico, utilidad metodológica y generación de datos sobre situaciones y experiencias de actores.

Probablemente, una de las mayores dificultades durante el aprendizaje radique en la propia interpretación situacional y el cómo, indicar posibles soluciones reconocidas.

Dado que, la educación universitaria en la contemporaneidad debe replantearse la incorporación de modelos de enseñanzas creativos para el éxito del aprendizaje (Jahnke et al., 2015; Nissim et al., 2016). En este estudio se consideró 6 indicadores que pueden medirse de forma sistemática para evaluar el aprendizaje durante la formación investigativa en docentes.
La búsqueda de resultados competitivos es esencial en la calidad de la formación universitaria (Huutoniemi \& Tapio, 2014) donde desafiar algunos paradigmas prevalecientes constituye un nuevo reto (Lotz et al., 2015) y fue en este estudio la pretensión, a través de indicadores conjugados de forma práctica con garantías de seguimiento.

O’Brien \& Sarkis (2014) indican la sostenibilidad de la educación, a partir de evitar la fragmentación de modelos establecidos y para ello, guiar cualquier orientación resulta necesaria. Considerar la implementación de criterios evaluativos que sean no solo dinámicos sino, modificables de acuerdo a, su carácter de notabilidad conduce a mejorar la enseńanza y por ende; la evaluación formativa del aprendizaje en docentes universitarios.

\section{REFERENCIAS BIBLIOGRÁFICAS}

Black, P. \& Wiliam, D. 2009. Developing the theory of formative assessment. Educational Assessment, Evaluation and Accountability, 21: 5-31.

Carless, D. 2011. From testing to productive student learning: Implementing formative assessment in Confucian-heritage settings. New York, NY: Routledge.

Cross, J. 2015. Metacognition in $\mathrm{L}_{2}$ listening: Clarifying instructional theory and practice. Tesol Quarterly, 49: 883-892.

Dimitrov, D.V. 2016. Medical internet of things and big data in healthcare. Healthcare Informatics Research, 22: 156-163. 
Facundo, M.L.; Landazábal, D.P.; Hernández, J.C.; Ruíz, Y. \& Vanegas, H. 2007. Visibilidad y formación en investigación. Estrategias para el desarrollo de competencias investigativas. Revista Studiositas, 2: 43-56.

Furtak, E.M. \& Ruiz-Primo, M.A. 2008. Making students' thinking explicit in writing and discussion: An analysis of formative assessment prompts. Science Education, 92: 799-824.

Gikandi, J.W.; Morrow, D. \& Davis, N.E. 2011. Online formative assessment in higher education: A review of the literature. Computers \& Education, 57: 2333-2351.

Huutoniemi, K. \& Tapio, P. 2014. Transdisciplinary sustainability studies: $A$ heuristic approach. New York: Routledge.

Iu, O.L.; Ungureanu, R. \& Rusu, C. 2014. European partnership for teacher training in Universities. Procedia - Social and Behavioral Sciences, 142: 200-206.

Jackson, D. 2016. Skill mastery and the formation of graduate identity in bachelor graduates: evidence from Australia. Studies in Higher Education, 41: 1313-1332.

Jahnke, I.; Haertel, T. \& Wildt, J. 2015. Teachers' conceptions of student creativity in higher education. Innovations in Education and Teaching International, 52: 1-9.

Lisle, J. 2015. Installing a system of performance standards for National Assessments in the Republic of Trinidad and Tobago: issues and challenges. Applied Measurement in Education, 28: 308-329.

Llamas-Nistal, M.; Fernández-Iglesias, M.J.; GonzálezTato, J.; Mikic-Fonte, F.A. 2013. Blended e-assessment: Migrating classical exams to the digital world. Computers \& Education, 62: 7287.

Lotz, S.H.; Walsisitka, A.E.J.; Kronlid, D. \& McGarry, D. 2015. Transformative, transgressive social learning: rethinking higher education pedagogy in times of systemic global dysfunction. Current Opinion in Environmental Sustainability, 16:73-80.
Messum, D.G.; Wilkes, L.M.; Jackson, D. \& Peters, K. 2016. Employability skills in Health Services Management: perceptions of recent graduates. Asia Pacific Journal of Health Management, 11: 25-32.

NRC (National Research Council). 2014. Developing assessments for the next generation science standards. Washington, DC: The National Academies Press.

Nissim, Y.; Weissblueth, E.; Scott-Webber, L. \& Amar, S. 2016. The effect of a stimulating learning environment on pre-service teachers' motivation and $21^{\text {st }}$ century skills. Journal of Education and Learning; 5: 29-39.

O'Brien, W. \& Sarkis, J. 2014. The potential of community-based sustainability projects for deep learning initiatives. Journal of Cleaner Production, 62: 48-61.

Sach, E. 2012. Teachers and testing: An investigation into teachers' perceptions of formative assessment. Educational Studies, 38: 261-276.

Suurtamm, C. \& Koch, M.J. 2014. Navigating dilemmas in transforming assessment practices: Experiences of mathematics teachers in Ontario, Canada. Educational Assessment, Evaluation and Accountability, 26: 263-287.

Tarighat, S. \& Khodabakhsh, S. 2016. Mobile-Assisted Language Assessment: Assessing speaking. Computers in Human Behavior, 64: 409-413.

Tejada, T.C.; Benítez, L.T. \& Ortiz, Á.V. 2008. Pedagogía para el desarrollo de competencias investigativas en los semilleros de investigación desde el inicio del pregrado. Revista Educación en Ingeniería, 3: 38-49.

Vo, M.H.; Zhu, C. \& Diep, A.N. 2017. The effect of blended learning on student performance at course-level in higher education: A metaanalysis. Studies in Educational Evaluation, 53, 17-28.

Yilmaz, Y. Ö.; Cakiroglu, J.; Ertepinar, H. \& Erduran, S. 2017. The pedagogy of argumentation in science education: science teachers' instructional practices, International Journal of Science Education, 39:1443-1464. 
Yin, X. \& Buck, G.A. 2015. There is another choice: An exploration of integrating formative assessment in a Chinese high school chemistry classroom through collaborative action research. Cultural Studies of Science Education, 10: 719-752.

Received December 2, 2018.

Accepted December 31, 2018. 